\title{
Influence of Heat Treatment on the Morphologies of Copper Nanoparticles Based Films by a Spin Coating Method
}

\author{
Wei Liu, ${ }^{1,2}$ Jiayu Guo, ${ }^{1}$ Zhonghua Fan, ${ }^{3}$ Xiaorong Wang, ${ }^{3}$ Dingkun Yu, \\ Rong Chen, ${ }^{3}$ Shisheng Huang, ${ }^{3}$ and Peng $\mathrm{He}^{2}$ \\ ${ }^{1}$ College of Materials Science and Engineering, China Jiliang University, Hangzhou 310018, China \\ ${ }^{2}$ State Key Laboratory of Advanced Welding and Joining, Harbin Institute of Technology, Harbin 150001, China \\ ${ }^{3}$ Hangzhou Huaguang Advanced Welding Materials Co., Ltd., Hangzhou 311112, China \\ Correspondence should be addressed to Peng He; hepeng@hit.edu.cn
}

Received 12 December 2016; Accepted 6 March 2017; Published 20 March 2017

Academic Editor: Xiaowei He

Copyright (C) 2017 Wei Liu et al. This is an open access article distributed under the Creative Commons Attribution License, which permits unrestricted use, distribution, and reproduction in any medium, provided the original work is properly cited.

\begin{abstract}
We have investigated the influence of heat treatment on the morphologies of copper nanoparticles based films on glass slides by a spin coating method. The experiments show that heat treatment can modify the sizes and morphologies of copper nanoparticles based films on glass slides. We suggest that through changing the parameters of heat treatment process may be helpful to vary the scattering and absorbing intensity of copper nanoparticles when used in energy harvesting/conversion and optical devices.
\end{abstract}

\section{Introduction}

Synthesis of metal nanoparticles with novel size-dependent physical and chemical and optical properties (e.g., quantumconfinement effects) plays a crucial role in many fields, such as optical label, catalysis, printed circuits, nanobrazing, and sensors during the past decade [1-5]. Among these various metal nanoparticles, noble metal nanoparticles such as $\mathrm{Au}$, $\mathrm{Ag}$, and Pt are getting special attention as they can be used in labels of immunoassays, lithographic fabrication, electronics, solar cell, nanobrazing, and surface enhanced spectroscope [6-10]. It is because they have large absorption and scattering coefficients. Furthermore, noble metal nanoparticles can be synthesized in different components, shapes (e.g., nanowire, nanodisk, nanorod, nanosphere, nanocube, and core-shell structure), and geometrical configurations, with which they can be self-assembled into different complex nanostructures required. Through these methods, the interaction between light and materials can be controlled and manipulated at a certain wavelength scale when they are used in energy harvesting/conversion and optical devices. Recently, a method is developed to enhance the efficiency and stabilize the emission spectrum in organic light-emitting diodes (OLEDs) using a light scattering layer fabricated with silver nanowires [11]. It is enhanced by $49.1 \%$ for the integrated external quantum efficiency. To reduce the usage of semiconductor materials, self-assembled gold nanorods are used to increase the photoconductivity in thin amorphous silicon semiconducting films. It is enhanced by a factor of 2 across the entire visible spectrum [12]. However, the high cost of noble metal nanoparticles has hindered their practical use.

In recent years, there are significant efforts to search cheap metal nanoparticles in order to displace noble metal nanoparticles such as $\mathrm{Au}, \mathrm{Ag}$, and $\mathrm{Pt}$, although the studies still have little research. Recently, aluminum nanoparticle and copper nanoparticles have attracted interest as alternative candidates. In order to enhance the solar absorption, a method is suggested to fabricate aluminum nanoparticles based structure using a porous template assisted self-assembly process [13]. It is demonstrated that the effective solar absorption can be over $96 \%$. However, the plasmon resonance wavelength of aluminum nanoparticles lies at the wavelength of around $150 \mathrm{~nm}$. In order to be used in energy harvesting/conversion devices, it should be shifted with changing the shape and geometrical configurations of aluminum nanoparticles. Due to its high oxidation activity, it is difficult to synthesize 
various shapes such as nanosphere, nanowire, and nanorod using a simple and cheap method. Copper nanoparticles are promising for these applications because of their considerably low cost compared to Ag, $\mathrm{Au}$, and $\mathrm{Pt}$, high electrical and thermal conductivities, and excellent resistance to ion migration. Furthermore, the plasmon resonance wavelength of copper nanoparticles lies in the visible wavelength region, similar to that of gold and silver nanoparticles. The scattering efficiency can be varied by changing shapes and geometrical configurations. Compared with the difficulty of synthesizing aluminum nanoparticles, various shapes and geometrical configurations of copper nanoparticles and copper based alloy nanoparticles have been synthesized by physical, chemical, and biological methods [2]. The study of various geometrical configurations and shapes has been conducted thoroughly and substantial progress has been made [14]. However, only little research has been focused on enhancing luminescence of laser dye or rare-earth by plasmonic coupling and scattering of copper nanoparticles $[15,16]$. In addition, there are many reports on preparing nanoparticles film using self-assembly technique (utilizing the chemical interaction between nanoparticles and the surface), lithography, and spin coating methods [17-19]. Among these methods, the spin coating method has getting attractions because self-assembly technique and lithography methods need a lot of procedures. Ag nanoparticle film is fabricated by the spin coating method and the sintering effect is investigated [20]. Unfortunately, there are few reports on preparing copper nanoparticles film using spin coating method.

In this paper, a simpler and cheaper method by the spin coating was used to fabricate copper nanoparticle based films on glass slides. Due to the importance of the shapes of copper nanoparticles when they are used in energy harvesting/conversion and optical devices, the influence of heat treatment on the sizes and morphologies of copper nanoparticles based film was investigated.

\section{Experimental Details}

2.1. Preparation of Copper Nanoparticles. Copper nanoparticles were synthesized by a mature polyol method in ambient atmosphere. Details of preparation process were described elsewhere [21]. The sodium hypophosphite monohydrate $\left(\mathrm{NaH}_{2} \mathrm{PO}_{2} \cdot \mathrm{H}_{2} \mathrm{O}\right)$, copper nitrate trihydrate $\left(\mathrm{Cu}\left(\mathrm{NO}_{3}\right)_{2} \cdot 3 \mathrm{H}_{2} \mathrm{O}\right)$, ethylene glycol, and polyvinylpyrrolidone (PVP, K29-32) were purchased from Aladdin Chemical Reagent Co. Ltd., P. R. China. The average molecular weight of the PVP polymer used was 58000. All the chemical regents were used as received. PVP was used to get an organic shell on the surface of copper nanoparticles for avoiding being oxidized. The following procedure was used to prepare copper nanoparticles. First, $4 \mathrm{~g} \mathrm{NaH} \mathrm{NO}_{2} \cdot \mathrm{H}_{2} \mathrm{O}$ and $2.5 \mathrm{~g}$ PVP were dissolved into $40 \mathrm{~mL}$ ethylene glycol at $90^{\circ} \mathrm{C}$. Then, $10 \mathrm{~mL}$ of $1 \mathrm{M}$ ethylene glycol solution of $\mathrm{Cu}\left(\mathrm{NO}_{3}\right)_{2} \cdot 3 \mathrm{H}_{2} \mathrm{O}$ at $90^{\circ} \mathrm{C}$ was injected into the above prepared solution of $\mathrm{NaH}_{2} \mathrm{PO}_{2} \cdot \mathrm{H}_{2} \mathrm{O}$ and PVP polymer under vigorously stirring.
The mixture solution was stirred for about 10 minutes and cooled down to room temperature. Finally, the prepared copper nanoparticles were separated by centrifugation, washed with absolute alcohol, and repeated for three times. After the above steps, it was well-dispersed in absolute alcohol again and the suspension can be stable for several weeks.

2.2. Preparation of Copper Nanoparticle Based Films. To get the copper nanoparticle based films on glass slides, the required concentration of copper nanoparticles in absolute alcohol $(0.4 \mathrm{~g} / 4 \mathrm{~g})$ was prepared under ultrasonic treatment for about 30 minutes. After setting well washed glass slides on the disk of the spin-coater machine, the solution of copper nanoparticles was dropped and spin-coated with 1000 rev. min $^{-1}$ for 12 seconds in air. Finally, the glass slides were kept in a tube furnace and annealed in a $5 \% \mathrm{H}_{2}-\mathrm{N}_{2}$ mix gas atmosphere for one hour at $100^{\circ} \mathrm{C}, 150^{\circ} \mathrm{C}, 200^{\circ} \mathrm{C}, 300^{\circ} \mathrm{C}$, and $400^{\circ} \mathrm{C}$, respectively.

2.3. Characterization of As-Prepared Copper Nanoparticles and Annealed Copper Nanoparticles Based Films. Thermogravimetric measurement (TG) was carried out by SMP/ PF7548/MET/600W thermogravimetric/differential thermal analyzer from the Swiss Mettler company. The heating rate is $10^{\circ} \mathrm{C} /$ minute of the heating rate with argon as a testing atmosphere. The size and morphology of copper nanoparticles was investigated by scanning electron microscopy (SEM) using a Hitachi SU8010 field-emission scanning electron microscope. X-ray diffraction (XRD) patterns were obtained on a D2 Phaser Benchtop XRD system (Bruker AXS, Germany).

\section{Results and Discussion}

To investigate the relation between the scattering and absorbing intensity with the size of copper nanoparticles, the extinction, scattering, and absorbing spectra of copper nanoparticles are simulated by Mie theory given by BHMIE code [22]. The Drude-Lorentz model for copper is obtained from by Ung and Sheng [23] The Drude-Lorentz parameters for copper are obtained from Rakić et al. [24]. The diameters of spherical nanoparticle considered are $120 \mathrm{~nm}$, $160 \mathrm{~nm}, 320 \mathrm{~nm}$, and $640 \mathrm{~nm}$, respectively. Figure 1 shows the extinction, scattering and absorbing spectra of copper nanoparticles with different diameters. It has been found that the tunability of extinction, scattering, and absorbing efficiency can be controlled effectively by change in diameters of copper nanoparticles. The methods of varying the diameters of copper nanoparticles here are heat treatment.

Figure 2 shows the XRD patterns of the as-prepared copper nanoparticles based films on glass slides. The results are in agreement with the values for a face-centered cubic crystal structure of copper phase (JCPDS number 04-0836). The peaks at $2 \theta=43.17^{\circ}, 50.33^{\circ}$, and $74.16^{\circ}$ correspond to (111), (200) and (220) Bragg reflections, respectively. No observed diffraction peaks are detected from any other impurities. There results identify that, under the protection 


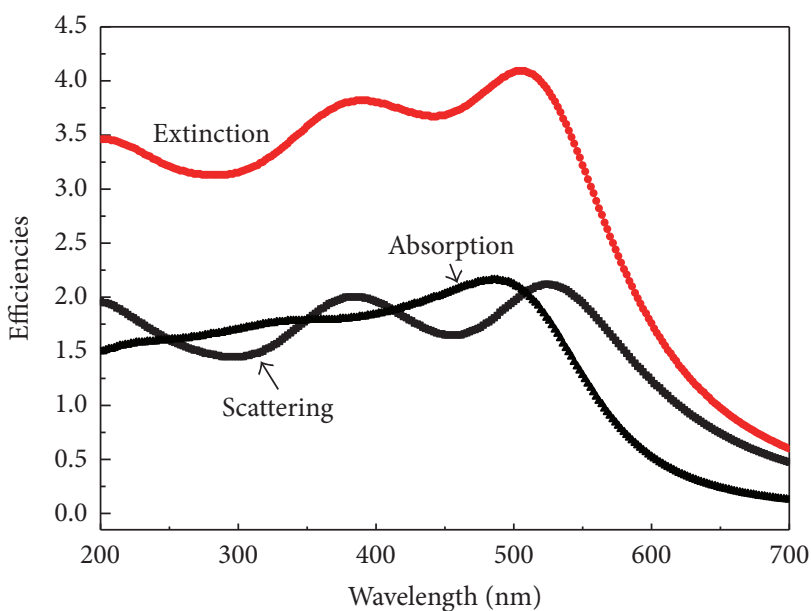

(a)

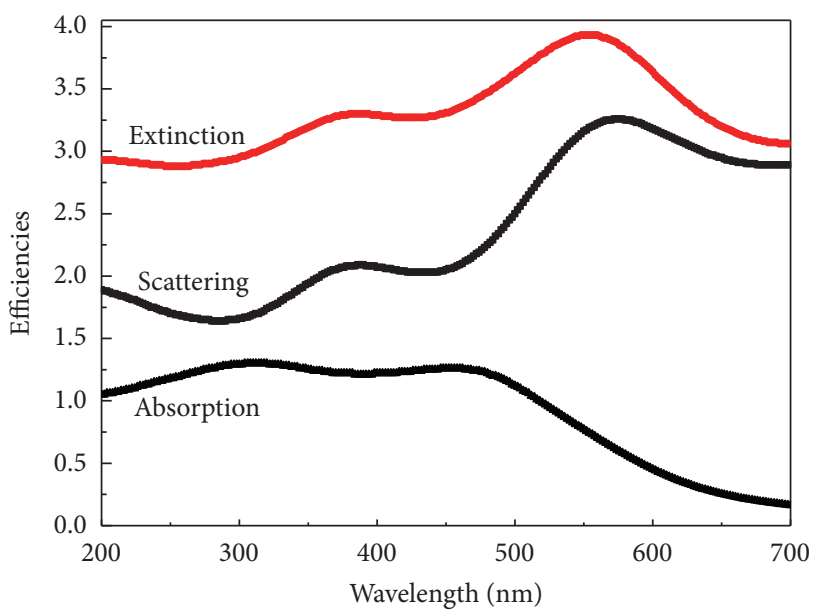

(c)

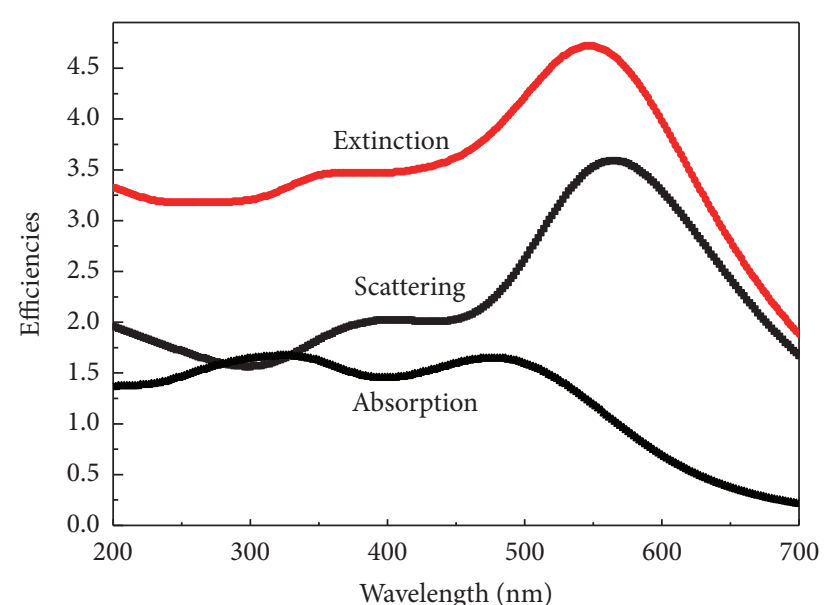

(b)

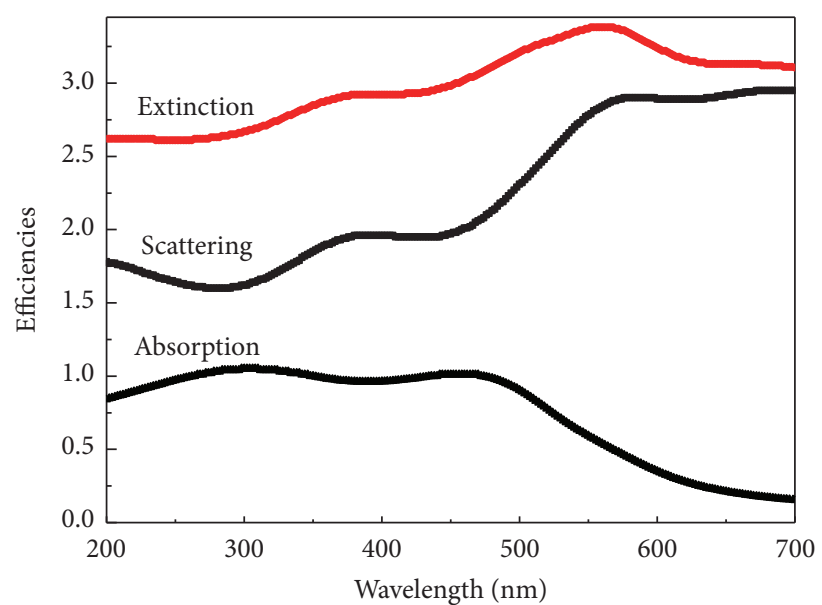

(d)

Figure 1: Calculated extinction, scattering, and absorption spectra of copper nanoparticles with different diameters. (a) $120 \mathrm{~nm}$, (b) $160 \mathrm{~nm}$, (c) $320 \mathrm{~nm}$, and (d) $640 \mathrm{~nm}$.

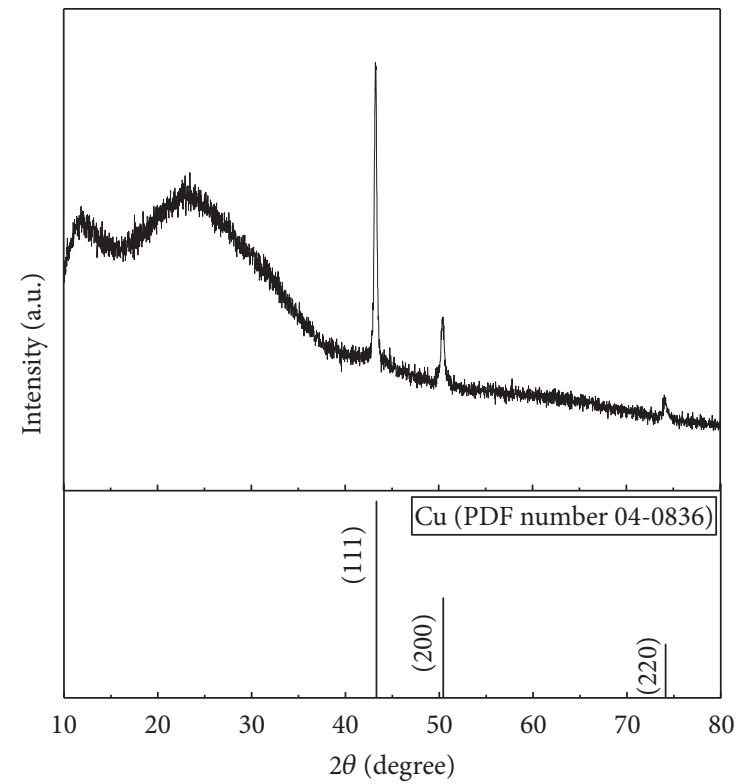

FIGURE 2: XRD diffraction patterns of the as-prepared copper nanoparticles based films on glass slides without anneal. by PVP polymer, the as-prepared copper nanoparticles are hardly oxidized into $\mathrm{Cu}_{2} \mathrm{O}, \mathrm{CuO}$, or $\mathrm{Cu}(\mathrm{OH})_{2}$. The sharp and strong diffraction peaks confirm that the as-prepared copper nanoparticles are highly crystalline.

TG analysis in an Ar atmosphere was used to study thermal decomposition behaviors of the as-prepared PVP coated copper nanoparticles, shown in Figure 3. An Ar atmosphere was used for TG measurement in order to prevent the oxidation of copper nanoparticles. The results indicate that the weight loss of $\sim 2.5 \%$, which could be attributed to small amount of absolute alcohol, ethylene glycol, and PVP polymer absorbed on the surface of as-prepared copper nanoparticles. The weight increases again at temperature over $600^{\circ} \mathrm{C}$, which is ascribed to the oxidation of $\mathrm{Cu}$ nanoparticles.

To investigate the oxidation protection performance of copper nanoparticles based films on glass slides, XRD patterns are measured at different heat treatment temperatures. According to TG analysis shown in Figure 3, the copper nanoparticles based films were annealed in a $5 \% \mathrm{H}_{2}-\mathrm{N}_{2}$ atmosphere for one hour at $100^{\circ} \mathrm{C}, 150^{\circ} \mathrm{C}, 200^{\circ} \mathrm{C}, 300^{\circ} \mathrm{C}$, and $400^{\circ} \mathrm{C}$, respectively. Considering the glass transition 


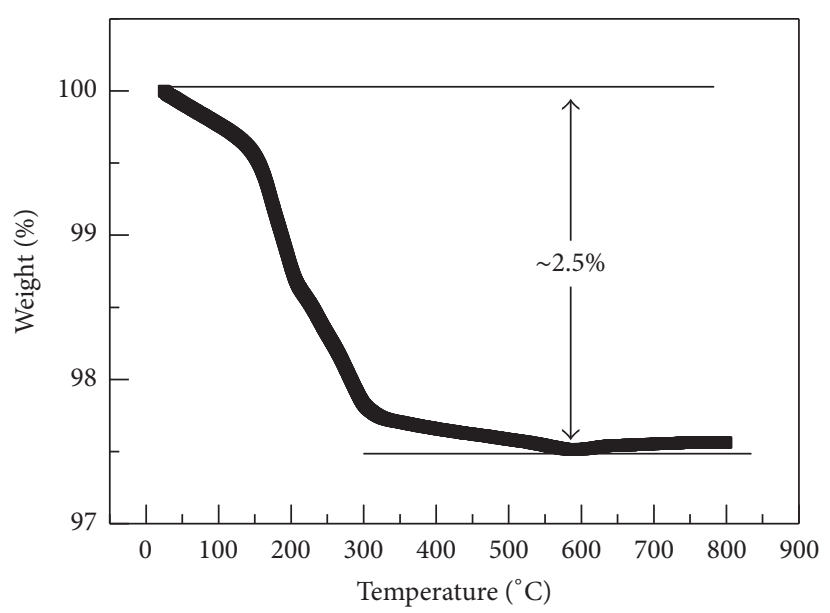

FIGURE 3: Thermal analysis of the as-prepared copper nanoparticles with argon as a testing atmosphere.

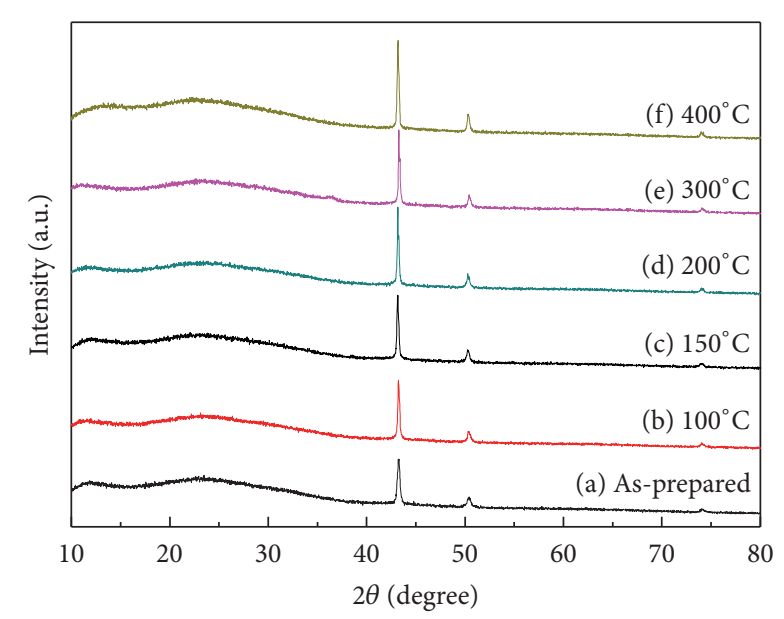

FIGURE 4: XRD diffraction patterns of the copper nanoparticles based films on glass slides annealed at different heat treatment temperatures by a spin coating method: (a) as-prepared, (b) $100^{\circ} \mathrm{C}$, (c) $150^{\circ} \mathrm{C}$, (d) $200^{\circ} \mathrm{C}$, (e) $300^{\circ} \mathrm{C}$, and (f) $400^{\circ} \mathrm{C}$.

temperature of glass slides used as substrates, the highest heat treatment temperature used is $400^{\circ} \mathrm{C}$. As shown in Figure 4, the intensity of the XRD peaks increases with heat treatment temperature from room temperature to $400^{\circ} \mathrm{C}$. These results show that the crystallizations of copper nanoparticles in the films become better. There are no observed characteristic peaks attributed to oxide impurities such as $\mathrm{Cu}_{2} \mathrm{O}, \mathrm{CuO}$, or $\mathrm{Cu}(\mathrm{OH})_{2}$ till $400^{\circ} \mathrm{C}$.

Due to the importance of the shapes and geometrical configurations of copper nanoparticles when used in energy harvesting/conversion and optical devices, it is significant to control the size and morphologies of copper nanoparticles. One of the simple methods to control them is changing the parameters of heat treatment process. Under heat treatment the residual solvent and organics are removed, leading to coalescence between adjacent copper nanoparticles. Figure 5 shows SEM images of the copper nanoparticles based films on glass slides. These results show that the copper nanoparticles pack to a multilayered structure. When heat treated at temperature under $200^{\circ} \mathrm{C}$, the morphologies of copper nanoparticles are still isolated nanoparticles, which are independent of heat treatment and the distributions of the size of copper nanoparticles are not uniform, shown in Figures 5(a)-5(c). The sintering process has not happened at these temperatures. However, when the heat treatment temperature increases to $200^{\circ} \mathrm{C}$, the copper nanoparticles start to melt as shown in Figure 5(d). As the heat treatment temperature increases to $300^{\circ} \mathrm{C}$, the size of copper nanoparticles increases obviously, as shown in Figure 5(e). When the heat treatment temperature increases to $400^{\circ} \mathrm{C}$, the size of copper nanoparticles continues to increase and some copper nanoparticles are interconnected, indicating that apparent sintering necks appeared. Furthermore, significant coarsening can be observed, shown in Figure 5(f).

\section{Conclusions}

We have reported on the influence of heat treatment on the sizes and morphologies of copper nanoparticles based films by a simple spin coating method. The heat treatment of copper nanoparticles based films on glass slide may be a promising method for modulating the sizes and morphologies of copper nanoparticles. When increasing the heat treatment temperature, the size keeps no obvious change while increasing at a critical heat treatment temperature. Significant coarsening of copper nanoparticles occurs when it continues increasing heat treatment temperature. Through this method it may be helpful to vary the scattering and absorbing intensity of copper nanoparticles within the entire visible spectrum.

\section{Conflicts of Interest}

The authors declare that they have no conflicts of interest. 


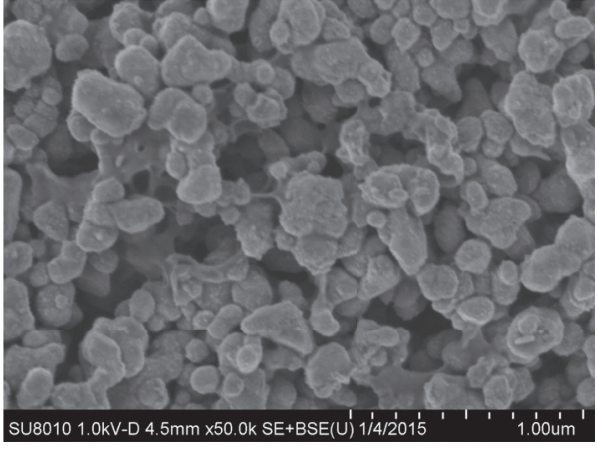

(a)

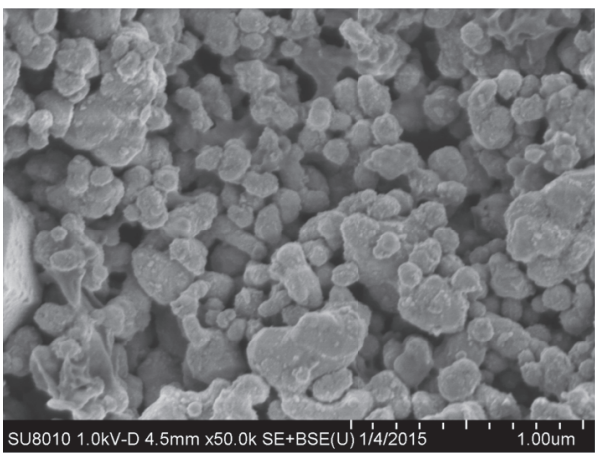

(c)

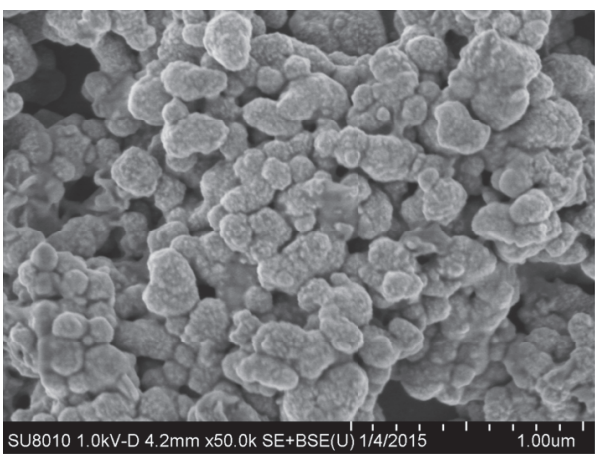

(e)

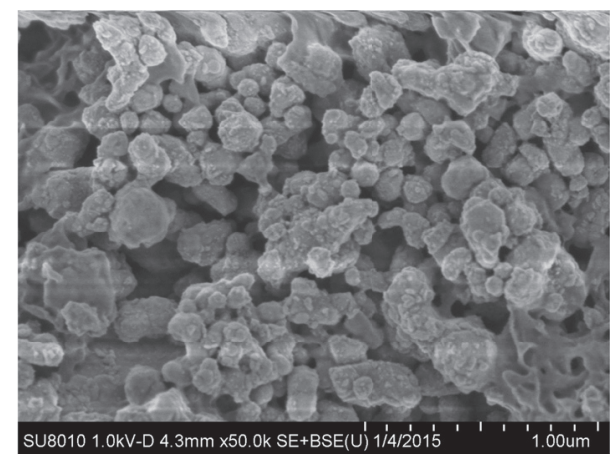

(b)

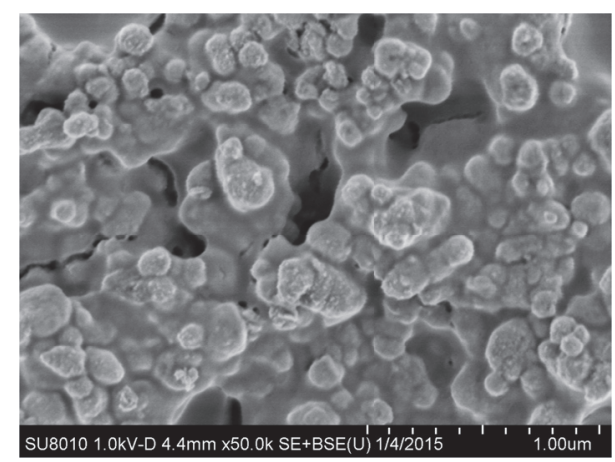

(d)

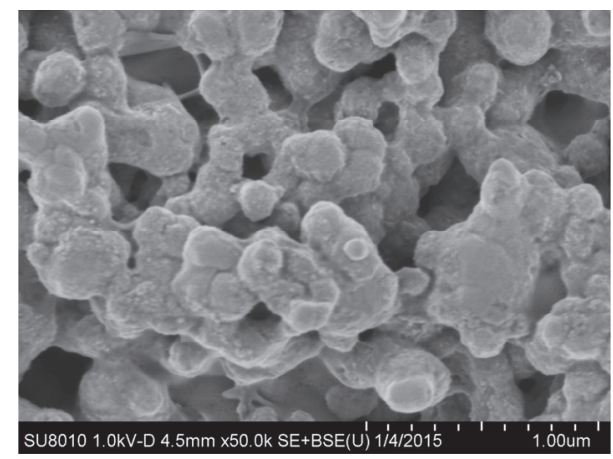

(f)

Figure 5: SEM images of the copper nanoparticles based films on glass slides at different heat treatment temperatures by a spin coating method: (a) as-prepared, (b) $100^{\circ} \mathrm{C}$, (c) $150^{\circ} \mathrm{C}$, (d) $200^{\circ} \mathrm{C}$, (e) $300^{\circ} \mathrm{C}$, and (f) $400^{\circ} \mathrm{C}$.

\section{Acknowledgments}

This work is partially supported by the Natural Science Foundation of Zhejiang (LY16F050005).

\section{References}

[1] S. Sarfraz, A. T. Garcia-Esparza, A. Jedidi, L. Cavallo, and K. Takanabe, "Cu-Sn bimetallic catalyst for selective aqueous electroreduction of $\mathrm{CO}_{2}$ to CO," ACS Catalysis, vol. 6, no. 5, pp. 2842-2851, 2016.

[2] M. B. Gawande, A. Goswami, F.-X. Felpin et al., " $\mathrm{Cu}$ and $\mathrm{Cu}-$ based nanoparticles: synthesis and applications in catalysis," Chemical Reviews, vol. 116, no. 6, pp. 3722-3811, 2016.

[3] K. J. Carroll, J. Ulises Reveles, M. D. Shultz, S. N. Khanna, and E. E. Carpenter, "Preparation of elemental $\mathrm{Cu}$ and $\mathrm{Ni}$ nanoparticles by the polyol method: an experimental and theoretical approach," Journal of Physical Chemistry C, vol. 115, no. 6, pp. 2656-2664, 2011.

[4] C. Schöttle, P. Bockstaller, D. Gerthsen, and C. Feldmann, "Tungsten nanoparticles from liquid-ammonia-based synthesis," Chemical Communications, vol. 50, no. 35, pp. 4547-4550, 2014.

[5] H. Shirai, M. T. Nguyen, Y. Ishida, and T. Yonezawa, "A new approach for additive-free room temperature sintering of conductive patterns using polymer-stabilized Sn nanoparticles," Journal of Materials Chemistry C, vol. 4, no. 11, pp. 2228-2234, 2016.

[6] X. Liu and D. Y. Lei, "Simultaneous excitation and emission enhancements in upconversion luminescence using plasmonic double-resonant gold nanorods," Scientific Reports, vol. 5, Article ID 15235, 2015. 
[7] J. Henzie, S. C. Andrews, X. Y. Ling, Z. Li, and P. Yang, "Oriented assembly of polyhedral plasmonic nanoparticle clusters," Proceedings of the National Academy of Sciences of the United States of America, vol. 110, no. 17, pp. 6640-6645, 2013.

[8] H. Zhang, M. Cao, W. Wu, H. Xu, S. Cheng, and L.-J. Fan, "Polyacrylonitrile/noble metal $\mathrm{SiO}_{2}$ nanofibers as substrates for the amplified detection of picomolar amounts of metal ions through Plasmon-enhanced fluorescence," Nanoscale, vol. 7, no. 4, pp. 1374-1382, 2015.

[9] D. D. Liana, B. Raguse, J. J. Gooding, and E. Chow, “Toward paper-based sensors: turning electrical signals into an optical readout system," ACS Applied Materials and Interfaces, vol. 7, no. 34, pp. 19201-19209, 2015.

[10] K. Suganuma, S. Sakamoto, N. Kagami, D. Wakuda, K.-S. Kim, and M. Nogi, "Low-temperature low-pressure die attach with hybrid silver particle paste," Microelectronics Reliability, vol. 52, no. 2, pp. 375-380, 2012.

[11] K. Lee, J.-W. Shin, J.-H. Park et al., "A light scattering layer for internal light extraction of organic light-emitting diodes based on silver nanowires," ACS Applied Materials and Interfaces, vol. 8, no. 27, pp. 17409-17415, 2016.

[12] C.-S. Chang and L. J. Rothberg, "Plasmon-enhanced photoconductivity in amorphous silicon thin films by use of thermally stable silica-coated gold nanorods," Chemistry of Materials, vol. 27, no. 9, pp. 3211-3215, 2015.

[13] L. Zhou, Y. Tan, J. Wang et al., "3D self-assembly of aluminium nanoparticles for plasmon-enhanced solar desalination," Nature Photonics, vol. 10, no. 6, pp. 393-398, 2016.

[14] S. Bhanushali, P. Ghosh, A. Ganesh, and W. Cheng, "1D copper nanostructures: progress, challenges and opportunities," Small, vol. 11, no. 11, pp. 1232-1252, 2015.

[15] R. Reisfeld, M. Grinberg, V. Levchenko et al., "Sol-gel glasses with enhanced luminescence of laser dye Rhodamine B due to plasmonic coupling by copper nanoparticles," Optical Materials, vol. 36, no. 10, pp. 1611-1615, 2014.

[16] H. K. Dan, D. Zhou, R. Wang et al., "Effect of Copper nanoparticles on the enhancement of upconversion in the $\mathrm{Tb}^{3+} / \mathrm{Yb}^{3+}$ co-doped transparent glass-ceramics," Optical Materials, vol. 39, pp. 160-166, 2015.

[17] Y.-K. Hong, H. Kim, G. Lee et al., "Controlled two-dimensional distribution of nanoparticles by spin-coating method," Applied Physics Letters, vol. 80, no. 5, pp. 844-846, 2002.

[18] H. Kodama, S. Momose, N. Ihara, T. Uzumaki, and A. Tanaka, "Disk substrate deposition techniques for monodisperse chemically synthesized FePt nanoparticle media," Applied Physics Letters, vol. 83, no. 25, pp. 5253-5255, 2003.

[19] S. Li, W. Li, Q. Liu et al., "Spin-coated Ag nanoparticles onto ITO substrates for efficient improvement of polymer solar cell performance," Journal of Materials Chemistry C, vol. 3, no. 6, pp. 1319-1324, 2015.

[20] K.-S. Chou, K.-C. Huang, and H.-H. Lee, "Fabrication and sintering effect on the morphologies and conductivity of nanoAg particle films by the spin coating method," Nanotechnology, vol. 16, no. 6, pp. 779-784, 2005.

[21] J. Yan, G. Zou, A. Hu, and Y. N. Zhou, "Preparation of PVP coated $\mathrm{Cu}$ NPs and the application for low-temperature bonding," Journal of Materials Chemistry, vol. 21, no. 40, pp. 15981-15986, 2011.

[22] C. F. Bohren and D. R. Huffman, Absorption and Scattering of Light by Small Particles, Wiley, New York, NY, USA, 1998.

[23] B. Ung and Y. Sheng, "Interference of surface waves in a metallic nanoslit," Optics Express, vol. 15, no. 3, pp. 1182-1190, 2007.
[24] A. D. Rakić, A. B. Djurišić, J. M. Elazar, and M. L. Majewski, "Optical properties of metallic films for vertical-cavity optoelectronic devices," Applied Optics, vol. 37, no. 22, pp. 5271-5283, 1998. 

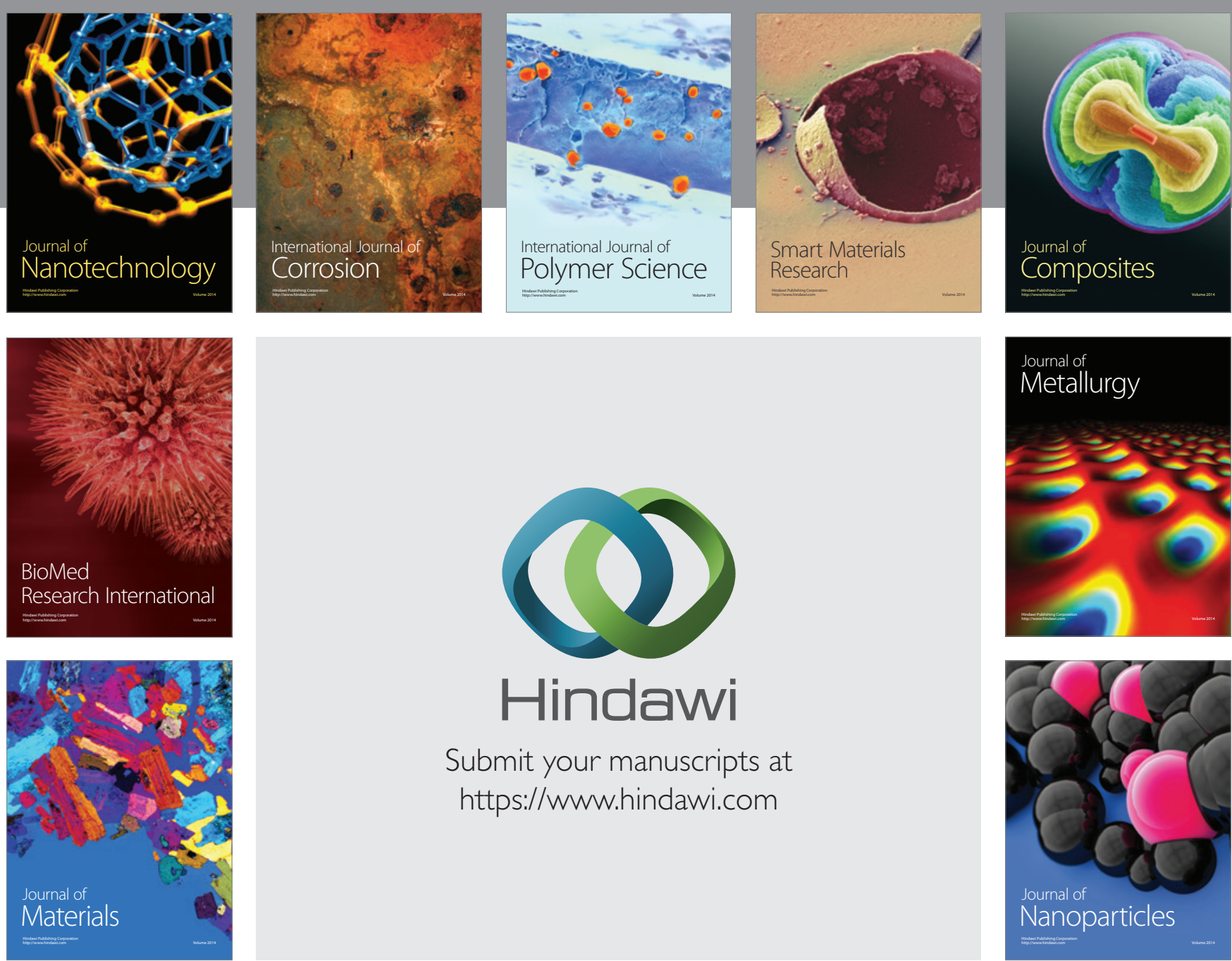

\section{Hindawi}

Submit your manuscripts at

https://www.hindawi.com

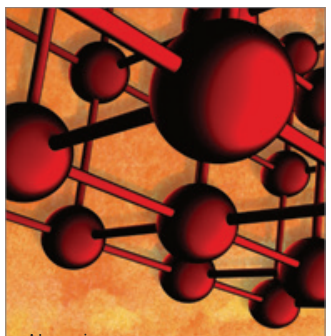

Materials Science and Engineering
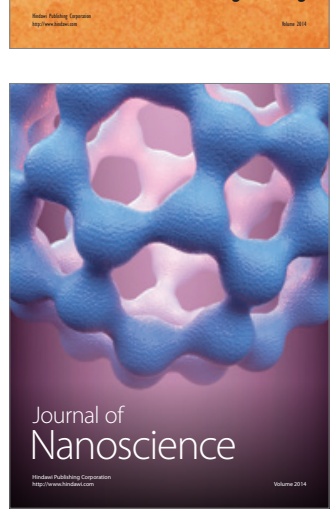
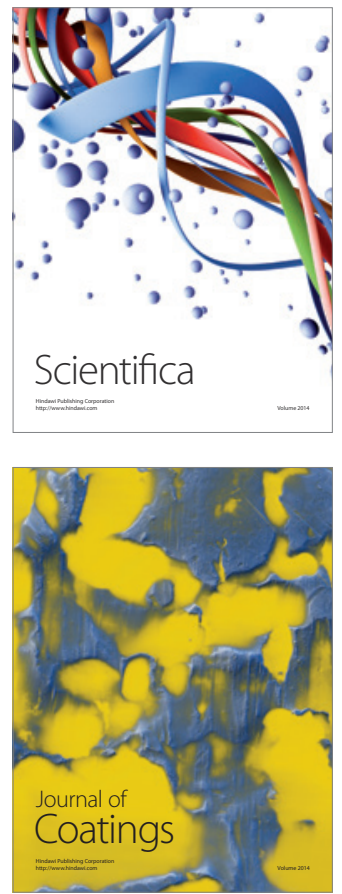
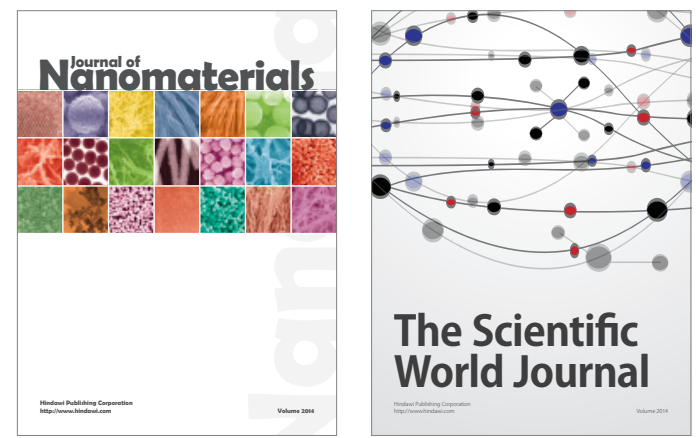

The Scientific World Journal
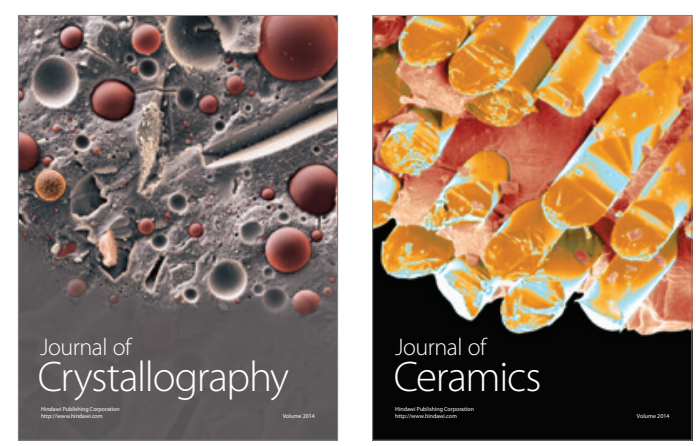
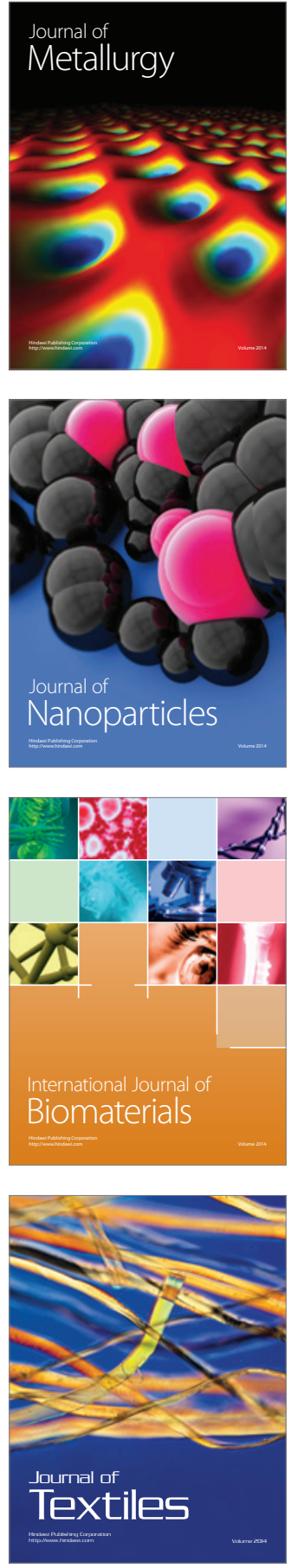